\title{
Targeted in vivo electroporation using nanoengineered microelectrodes
}

\author{
Daniel SchwarZ ${ }^{1.3}$ Andreas T. Schaefer ${ }^{1,3.5}$ \\ Behavioural Neurophysiology, Max-Planck-Institute for Medical Research, Heidelberg, Germany \\ Department of Neuroradiology, Heidelberg University Hospital, Germany \\ ${ }^{3}$ Department of Anatomy and Cell Biology, Faculty of Medicine, University of Heidelberg, Germany \\ ${ }^{+}$The Francis Crick Institute, Neurophysiology of Behaviour Laboratory, London, UK \\ ${ }^{5}$ Department of Neuroscience, Physiology \& Pharmacology, University College London, UK
}

Present addresses:

*Daniel Schwarz, Department of Neuroradiology, Heidelberg University Hospital, Im Neuenheimer Feld 400, 69120 Heidelberg, Germany; Tel: +49-6221-56-39392, Fax: +49-6221-56-4673, email: daniel.schwarz@med.uniheidelberg.de,

*Andreas T. Schaefer, Neurophysiology of Behaviour Laboratory, Francis Crick Institute, 1 Midland Road, London NW1 1AT, United Kingdom; Tel: +44 203796 1579, andreas.schaefer@crick.ac.uk

\section{Abstract/Summary:}

Targeted electroporation by using glass microelectrodes is a popular and versatile tool allowing for easy manipulation of single cells and cell ensembles in living tissue. Because of the highly focal distribution of the electric field, however, the range of reversible electroporation without causing irreversible damage is tight - especially when aiming for larger electroporation volumes. In this chapter, we describe the production of nanoengineered electroporation microelectrodes (NEMs), a practicable way to prepare glass microelectrodes providing a more even distribution around the tip of a pipette by using nanotechnological methods.

Keywords: Electroporation, nanoengineering, microelectrodes, in vivo 


\section{1) Introduction:}

The technique of targeted electroporation using a glass microelectrode was popularized by Haas et. al in 2001 to transfect single neuronal cells of the tadpole in vivo [1]. They employed fine needle glass microelectrodes as normally used for patch-clamp recordings to deliver the electric field. This versatile approach greatly broadened the in vivo applicability of electroporation because it proved that highly inhomogeneous electric fields with a distant grounding electrode could effectively be employed for the purpose and that plate or array electrodes were not necessarily required. In subsequent years, this approach has increasingly been used especially in the neurosciences and has also been shown to be effective beyond the single cell level [2-5]. An important limitation, however, comes with the highly focal electric field distribution of such glass microelectrodes: Because of the small pipette-tip of $\sim 1 \mu \mathrm{m}$ in diameter, the electric peak potentials centered to the tip rise rapidly with increasing stimulation intensity of the system [6]. This is a major drawback when the technique is intended for larger electroporation volumes as transmembrane potentials beyond $700 \mathrm{mV}$ are thought to be responsible for irreversible damage and lysis of many cellular structures [7].

In this chapter, we describe an approach to handling this issue by nanotechnological modification of the pipette thus creating a more homogeneous electric field distribution around the tip $[6,8]$ thus leading to higher stimulation intensities with greater electroporation volumes at reduced peak potential regions (Fig. 1).

\section{2) Materials:}

\section{Fabrication of NEMs:}

1. Standard thin-walled borosilicate glass (outer diameter $2.0 \mathrm{~mm}$, inner diameter $1.7 \mathrm{~mm}$, Science Products GmbH, Hofheim, Germany)

2. Sutter P-2000 laser puller or Flaming/Brown type P-97 micropipette puller (Sutter Instruments, Novato, CA, USA)

1. Gold wire (diameter $0.2 \mathrm{~mm}$, Plano GmbH, Wetzlar, Germany)

2. Conductive silver paint (SPI, Structure Probe Inc., West Chester, PA, USA)

3. High-vacuum sputtering and coating system (Bal-tec MED 020, Leica, Wetzlar, Germany) (see Note 1) 
4. Combined Focused-ion-beam (FIB) - Scanning electron microscope (SEM) workstation (Neon 40EsB, Zeiss, Oberkochen, Germany)

\section{In vivo electroporation experiments}

1. 6- to 8-week-old MOR174-9 mice [9] are used for this procedure, maintained under appropriate animal welfare guidelines and approved by the responsible Institutional Animal Care and Use Committee (see Note 2)

2. Microsurgical equipment including a surgical microscope (Leica microsystems, Wetzlar, Germany)

3. Heating pad connected to a feedback circuit provided by a rectal probe (FHC-40908, BASi Corporation, West Lafayette, IN, USA)

4. Instant adhesive Loctite 4011 (Loctite Corporation, Rocky Hill, CT, Mississauga, Ontario)

5. Paladur dental acrylic (Heraeus Holding GmbH, Hanau, Germany)

6. Dental drill

7. Sharp and small needle tip

8. Flamed tungsten wire

9. Two-photon microscope setup equipped with a Ti-Sapphire laser (Coherent, Dieburg, Germany) including a 16x water immersion objective (0.8 NA, Nikon)

10. Extracellular Ringer's solution ( $\mathrm{NaCl} 135.0 \mathrm{mM}, \mathrm{KCl} 5.4 \mathrm{mM}$, Hepes $5.0 \mathrm{mM}, \mathrm{MgCl}_{2}$ $1.0 \mathrm{mM}, \mathrm{CaCl}_{2} 2.0 \mathrm{mM}, \mathrm{pH} 7.2,280 \mathrm{mOsm} / \mathrm{kg}$ )

11. Tetramethylrhodamine (TMR) or Fluorescein 3000 MW dextran-conjugated Dyes (both Invitrogen, Life Technologies $\mathrm{GmbH}$ )

12. Micromanipulator (Luigs\&Neumann, Ratingen, Germany)

13. Stimulus isolation unit (ISO-STIM 01D, npi electronic GmbH, Tamm, Germany)

14. Paraformaldehyde solution (4\%, $\mathrm{pH}$ adjusted to 8.9)

\section{3) Methods:}

\section{Fabrication of NEMs:}

1. Pull glass capillaries to a long shank as to ensure better applicability in later in vivo experiments. Final tip size should be 3.5 to $4.5 \mu \mathrm{m}$ in diameter (see Note 3 and 4 ). 
2. Place glass pipettes in the high-vacuum sputtering and coating system in an upright manner and heat-coat twice for 40 seconds with a piece of gold wire. The pipette need to be placed close to the heating coil. This procedure ensures an even deposition of the gold layer on the surface of the pipette tip. Finally, a layer thickness of approximately $100-200 \mathrm{~nm}$ should be reached. Pipettes with a thicker gold coating or a rough surface should be discarded.

3. Paint pipettes with an additional layer of conductive silver paint along the shaft in order to obtain sufficient electric conductivity of the surface to prevent pipettes from overcharging during application of FIB-mode and to provide better imaging quality in subsequent imaging steps using SEM-mode.

4. After this pre-treatment, coated and painted glass micropipettes are put in the combined FIB-SEM workstation which is equipped with a gallium ion source for FIB-mode milling purposes $[10,11]$. We suggest using the gallium-ion gun an angle of $90^{\circ}$ to the principal axis of the pipette to mill 5 square-shaped holes into the glass wall along the shaft, up to an axial distance of $25 \mu \mathrm{m}$ from the tip; each set apart by $5 \mu \mathrm{m}$. Size of individual holes needs to decrease with increasing distance to the tip to account for the axial resistance of the pipette (see below for more detail). Milling current und dwell time per area needs to be chosen such that every single shot can penetrate both glass walls leaving behind two openings on opposite sides of the pipette. This is typically achieved after 1-2 seconds for small holes and after 10-15 seconds fort larger holes. Next, pipettes are rotated axially by 90 degrees and the procedure is repeated. Thus, finally, the cone will assume a 5-level-cross-like pattern having the largest openings close to the tip (Figure 2).

\section{Estimation of the internal electrical architecture of NEMs}

1. Basic concept of the internal electrical NEM architecture is to take advantage of the axial resistance as a current divider.

2. To estimate the current distribution, it is necessary to calculate each individual resistor which follows Pouillet's law in case of an electrolyte-filled capillary (see Note 5).

a. For the tip-end region of a pulled glass capillary, 'the basic resistor', a uniformly conical shape with the included angle Theta $(\theta)$ is assumed. Taking these geometrical constraints into account the following expression is obtained for calculation of the total resistance $R_{\text {Tip }},[12,13]$ : 


$$
R_{\text {Tip }}=\rho \frac{\cot \left(\frac{\theta}{2}\right)}{\pi}\left(\frac{1}{r_{\text {tip }}}-\frac{1}{r_{x}}\right)
$$

where $r_{\text {tip }}$ is the radius at the tip of the pipette and $r_{x}$ the basis of the cone and $\rho$ is the specific electrical resistivity.

b. The same principles according to Pouillet apply to the resistance of every inserted hole, with the difference that the geometrical shape is not conical but an approximated cuboid with specified edge length $s$ and depth $d$. Assuming that each 'level' consists of four holes of the same electrical properties and distance to the pipette-tip, each level can be treated as one resistor $\left(R_{\text {level }}\right)$ :

$$
R_{\text {level }}=\rho \frac{d}{4 s_{\text {level }}^{2}} .
$$

3. Tip geometry, size of each hole and number of holes represent free parameters with direct impact on the resistance and certainly a multitude of solutions for the current distribution can be thought of and depend upon one's specific experimental needs. However, in general it is important that distant holes to the pipette tip need to be small because of the higher axial resistance downstream while holes in vicinity to the tip should be larger (see Note 6). We found a design to work best for us [6] with the following level-specific edge lengths: $s_{1}=2.12 \mu \mathrm{m}, s_{2}=1.65 \mu \mathrm{m}, s_{3}=1.25 \mu \mathrm{m}, s_{4}=$ $0.82 \mu \mathrm{m}$ and $s_{5}=0.6 \mu \mathrm{m}$.

\section{In vivo electroporation experiments}

1. Animals are anaesthetized by intraperitoneal injection of meditomidin $0.75 \mathrm{mg} / \mathrm{kg}$, fentanyl $0.025 \mathrm{mg} / \mathrm{kg}$ and midazolam $10.00 \mathrm{mg} / \mathrm{kg}$ after brief inhalative isofluranenarcosis. The animal's body temperature should be kept at $37^{\circ} \mathrm{C}$ throughout the whole procedure.

2. Keep the head tightly between two metal rods being placed in the external acoustic meatus on either side. After removing the scalp and clean exposure of the cranial bone, the periosteum is thoroughly removed using a scalpel. Next, a metal head-plate is attached around the brain area of interest using instant adhesive.

3. On top of the head-plate, a sealing well is formed out of dental acrylic around the region of interest. This is needed for later water immersion imaging. Additionally, a grounding electrode of chlorided silver wire is inserted in one of the walls.

4. On a different holder which holds the head-plate - and thus the head - tightly in place the skull overlaying the brain area of interest is thinned using the dental drill. The final 
bone lamella is removed by using a very sharp and small needle tip. To prevent breaking or clogging of the pipette, the dura mater needs to be carefully peeled away using a fine flamed tungsten wire.

5. Subsequently, mice are placed under a two-photon microscope [14] and the sealed well is filled with the extracellular Ringer's solution. For exact pipette targeting, a 16x water immersion objective should be used. To achieve two-photon excitation, the laser is tuned to the appropriate wave-length (e.g. $880 \mathrm{~nm}$ in case of GFP).

6. An NEM is tip-filled with the extracellular Ringer's solution containing either TMR $(8.3 \mathrm{mg} / \mathrm{ml})$ or Fluorescein $(12.5 \mathrm{mg} / \mathrm{ml}) 3000 \mathrm{MW}$ dextran-conjugated Dyes. Complementary backfilling with plain Ringer's as described.

7. The pipette is then mounted on the micromanipulator and the grounding electrode of the well connected to the ground of the stimulus isolation unit. Then place the tip of the pipette to the target structure of interest under two-photon guidance and start the electroporation program (see Note 7).

8. Standard electroporation settings are adapted from current literature on glass microelectrode electroporation [2-4,9] and imply $25 \mathrm{~ms}$ square pulses delivered at a frequency of $2 \mathrm{~Hz}$ over $5 \mathrm{~min}$ at a stimulation intensity of $50 \mu \mathrm{A}$ per pulse.

9. $30-60 \mathrm{~min}$ after completion of the electroporation protocol, animals are sacrificed by a transcardial paraformaldehyde perfusion (4\%, $\mathrm{pH}$ adjusted to 8.9 ) and the brain is removed for further histological workup.

\section{4) Notes:}

1. While this is still a tool not common in neurophysiology departments, FIB milling systems are standard tools in any engineering department and increasingly used for neuroanatomical studies as well [15]

2. Depending on the exact target and/or brain region of interest, any other genetic background strain can be used. Expression of a fluorescent protein in a target structure facilitates accurate localization for in vivo electroporation.

3. Since current distribution along the pipette tip critically depends on the exact geometry of the cone, shallow cone angles are preferred for a more even distribution. This is achieved more easily with a thin-walled glass capillary as compared to a thick-walled one. 
4. Also, a wider tip opening will favor a more even distribution. This, however, may come at the cost of more tissue damage so that we found a tip opening of 3.5 to $4.5 \mu \mathrm{m}$ to be a reasonable compromise.

5. For simplification, a uniform flow of electric current, a constant specific resistance $\rho$ and no conductivity through the wall of the glass are assumed in this description.

6. In theory, hole resistance could also be changed by the depth parameter $\mathrm{d}$. This, however, would either require the deposition of material or to carve out a shallow around a hole which would make the fabrication process much more complicated and lengthy.

7. During tissue insertion, permanent low positive pressure $(10-20$ mbar $)$ is applied to the pipette to allow constant dye-perfusion of the tissue and to prevent tissue from extending into the holes of the pipette.

\section{References:}

1. Haas K, Sin WC, Javaherian A, Li Z, Cline HT (2001) Single-cell electroporation for gene transfer in vivo. Neuron 29 (3):583-591. doi:S0896-6273(01)00235-5 [pii]

2. Nagayama S, Zeng S, Xiong W, Fletcher ML, Masurkar AV, Davis DJ, Pieribone VA, Chen WR (2007) In vivo simultaneous tracing and $\mathrm{Ca}(2+)$ imaging of local neuronal circuits. Neuron 53 (6):789-803. doi:S0896-6273(07)00138-9 [pii] 10.1016/j.neuron.2007.02.018

3. Nevian T, Helmchen F (2007) Calcium indicator loading of neurons using single-cell electroporation. Pflugers Arch 454 (4):675-688. doi:10.1007/s00424-007-0234-2

4. Hovis KR, Padmanabhan K, Urban NN (2010) A simple method of in vitro electroporation allows visualization, recording, and calcium imaging of local neuronal circuits. J Neurosci Methods 191 (1):1-10. doi:DOI 10.1016/j.jneumeth.2010.05.017

5. Ke MT, Fujimoto S, Imai T (2013) SeeDB: a simple and morphology-preserving optical clearing agent for neuronal circuit reconstruction. Nat Neurosci 16 (8):1154-1161. doi: $10.1038 / \mathrm{nn} .3447$

6. Schwarz D, Kollo M, Bosch C, Feinauer C, Whiteley I, Margrie TW, Cutforth T, Schaefer AT (2018) Architecture of a mammalian glomerular domain revealed by novel volume electroporation using nanoengineered microelectrodes. Nature communications 9 (1):183. doi:10.1038/s41467-017-02560-7

7. Sale AJH, Hamilton WA (1968) Effects of High Electric Fields on Micro-Organisms .3. Lysis of Erythrocytes and Protoplasts. Biochim Biophys Acta 163 (1):37-43

8. Carraro T, Dorsam S, Frei S, Schwarz D (2018) An Adaptive Newton Algorithm for Optimal Control Problems with Application to Optimal Electrode Design. J Optimiz Theory App 177 (2):498-534. doi:10.1007/s10957-018-1242-4

9. Sosulski DL, Bloom ML, Cutforth T, Axel R, Datta SR (2011) Distinct representations of olfactory information in different cortical centres. Nature 472 (7342):213-216. doi:nature09868 [pii] 10.1038/nature09868 
10. Vasile MJ, Nassar R, Xie J, Guo H (1999) Microfabrication techniques using focused ion beams and emergent applications. Micron 30 (3):235-244

11. Langford RM (2006) Focused ion beams techniques for nanomaterials characterization. Microsc Res Tech 69 (7):538-549. doi:10.1002/jemt.20324

12. Snell FM (1969) Some electrical properties of fine-tipped pipette microelectrodes. In: Lavallee M, Schanne OF, Hebert NC (eds) Glass microelectrodes. John Wiley \& Sons, United States of America, pp 111-123

13. Sakmann B, Neher E (1995) Geometric parameters of pipettes and membrane patches. In: Sakmann B, Neher E (eds) Single-channel recording. Second edition edn. Plenum Press, New York, NY, USA, pp 637-650

14. Denk W, Strickler JH, Webb WW (1990) 2-Photon Laser Scanning Fluorescence Microscopy. Science 248 (4951):73-76

15. Briggman KL, Bock DD (2012) Volume electron microscopy for neuronal circuit reconstruction. Curr Opin Neurobiol 22 (1):154-161. doi:10.1016/j.conb.2011.10.022

\section{Figures/Figure legends:}

Figure 1: Comparison of the finite element model (FEM) of an NEM and a standard glass electrode

Centre-cut of a 3D FEM illustrating the assumed total effective electroporation volume $>200$ $\mathrm{mV}$ and its distribution around the pipette tip at $50 \mu \mathrm{A}$ employing an NEM (a) and a standard glass (b) micropipette (white, $\mathrm{V}_{\mathrm{m}}=$ transmembrane potential). Volume elements exceeding a transmembrane potential of $700 \mathrm{mV}$ are coloured in red. The volume beyond $700 \mathrm{mV}$ is markedly smaller in case of the NEM when compared to a standard pipette ( $20.4 \mu \mathrm{m}^{3} \mathrm{vs} .220 .1$ $\left.\mu \mathrm{m}^{3}\right)$.

\section{Figure 2: Fabrication of NEMs [adapted from [6]]}

a) Workflow of the pipette fabrication process: After pulling long-tapered patch-clamp-like pipettes (1), pipettes are successively coated with a thin gold layer (2) and a conductive silver paint (3) before FIB-assisted milling can be performed (4). b) Inside camera view of the vacuum chamber for SEM-imaging and FIB-assisted milling. Glass pipettes are mounted and aligned at an angle of $90^{\circ}$ relative to the principal axis of the FIB beam. Gallium-ion gun shown in the right upper corner, electron beam mounted vertically in the central portion of the chamber. c) Example micropipette after successful insertion of the five-level hole design as seen in the high resolution FIB-imaging mode. 

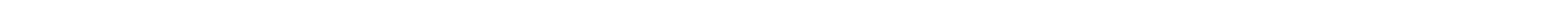

$5 \mu \mathrm{m}$ $V_{m}<200 \mathrm{mV}$
$V_{m}>700 \mathrm{mV}$

$5 \mu \mathrm{m}$

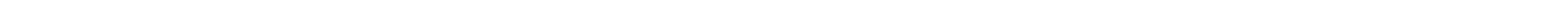


